\title{
DAMPening inflammation
}

Immune cells use evolutionarily conserved pattern recognition receptors (PRRs) to trigger an inflammatory response to both infection and cellular damage. But how can a common set of receptors be used to discriminate between pathogenassociated molecular patterns (PAMPs) of foreign microorganisms and danger-associated molecular patterns (DAMPs) released from dying cells to ensure that the response is appropriate to the level of threat? New research has identified a signalling pathway that restricts the response to DAMPs but not PAMPs.

In an acetaminophen (AAP)induced model of liver necrosis, Chen et al. showed that a dose of AAP that is sublethal for wild-type mice caused rapid death in mice deficient for $\mathrm{CD} 24$, which coincided with a large increase in the production of the pro-inflammatory cytokines interleukin-6 (IL-6), tumour necrosis factor (TNF) and CC-chemokine ligand 2 (CCL2). The CD24-deficient mice also had increased serum levels of alanine transaminase (ALT; which is an indicator of liver damage), liver haemorrhage and liver necrosis. Together, the results indicate that CD24 protects mice against inflammation-induced liver damage triggered by AAP.

To explain this effect, the authors looked for new ligands for CD24 and found that high mobility group box 1 (HMGB1) - a prototypical DAMP that is released from necrotic cells interacts specifically and directly with CD24. Antibody-mediated blockade of HMGB1 rescued AAP-treated CD24-deficient mice from death and was associated with decreased levels of ALT, IL-6, TNF and CCL2. The anti-inflammatory effect of CD24 on the response to HMGB1 signalling through its PRR was shown to result from binding of CD24 to human sialic acid-binding immunoglobulinlike lectin 10 (Siglec10), which is thought to be a negative regulator of nuclear factor- $\kappa \mathrm{B}(\mathrm{NF}-\kappa \mathrm{B})$ signalling through its cytoplasmic immunoreceptor tyrosine-based inhibitory motifs. Siglec 10 also bound to HMGB1 in a CD24-dependent

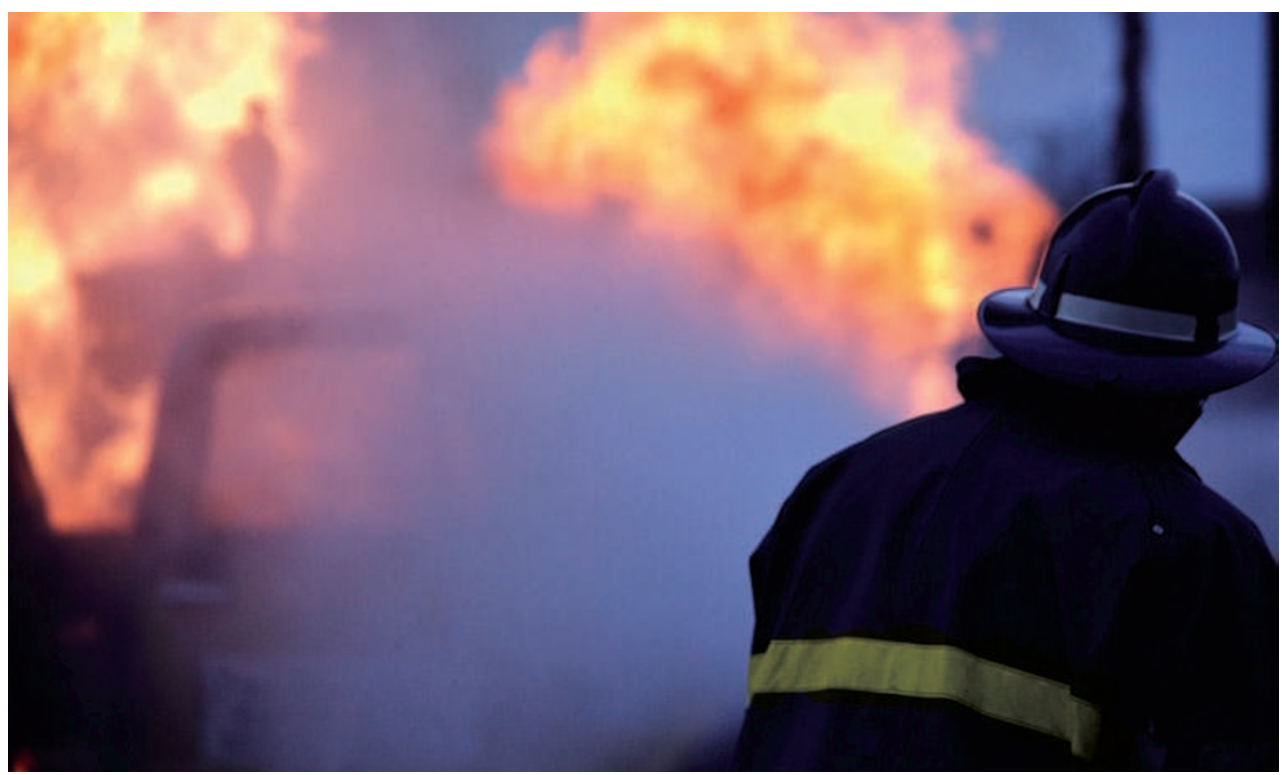

manner, indicating that a trimolecular complex is formed. Mice deficient for SiglecG (the probable mouse homologue of human Siglec10) had an increased inflammatory response and greater sensitivity (75\% death rate) to a sublethal dose of AAP, which could be inhibited by HMGB1-specific antibodies.

Both CD24 and SiglecG are expressed by dendritic cells (DCs), which are also known to respond to HMGB1. Stimulation of CD24- or SiglecG-deficient DCs with HMGB1 resulted in increased nuclear translocation of the NF- $\kappa B$ subunit $\mathrm{p} 65$ and significantly greater production of IL-6 and TNF compared with wildtype DCs. Importantly, deficiency of CD24 or SiglecG did not affect the DC response to the microbial PAMP lipopolysaccharide (LPS) in terms of either NF- $\mathrm{B}$ nuclear translocation or inflammatory cytokine production, and CD24- or SiglecG-deficient mice had wild-type death kinetics in response to a lethal dose of LPS.

These results indicate that the CD24-SiglecG pathway selectively represses the NF- $\kappa \mathrm{B}$-driven inflammatory response to the nuclear DAMP HMGB1 but not to the microbial PAMP LPS. The authors also show that SiglecG associates with the heat-shock proteins HSP70 and HSP90 in a CD24-dependent manner and inhibits the inflammatory response to these cytoplasmic DAMPs. So, CD24-SiglecG signalling might be a general mechanism to distinguish between tissue injury and infection and prevent an 'overzealous' response to damaged tissue.

Kirsty Minton

ORIGINAL RESEARCH PAPER Chen, G.-Y. et al. CD24 and Siglec-10 selectively repress tissue damage-induced immune responses. Science 5 Mar 2009 (doi:10.1126/science.1168988) FURTHER READING Kono, H. \& Rock, K. L. How dying cells alert the immune system to danger. Nature Rev. Immunol. 8, 279-289 (2008) 